\title{
Utilización de la investigación por gestores de salud: desarrollo y validación de una herramienta de autodiagnóstico
}

\author{
Miguel Ángel González-Block BA, MA, D Sci, (') Francisco Javier Mercado, D Sci,(2) \\ Héctor Ochoa, MD, PhD, ${ }^{(3)}$ Héctor Rivera MD, ${ }^{(4)}$ Álvaro J Idrovo, D Sci. ${ }^{(1)}$
}

González-Block MA, Mercado FJ, Ochoa H, Rivera H, Idrovo AJ. Utilización de la investigación por gestores de salud: desarrollo y validación de una herramienta de autodiagnóstico. Salud Publica Mex 2008;50:490-497.

\section{Resumen}

Objetivo. Adaptar y evaluar una herramienta de autodiagnóstico de la capacidad de los equipos de gestión de salud para utilizar la investigación, con énfasis en su adquisición, análisis, adaptación y aplicación. Material y métodos. La herramienta se tradujo al español y se elaboró un manual sometido a la autorización de la Comisión de Ética del Instituto Nacional de Salud Pública, antes de su aplicación durante el periodo de abril a noviembre del 2007 en 122 unidades (tasa de respuesta de $84.4 \%$ ) para cubrir una amplia gama de instituciones en seis entidades contrastantes de México, según el índice de desarrollo humano (IDH). Se validó la herramienta de manera interna y externa con métodos estadísticos. Resultados. Se observaron diferencias en la capacidad de los equipos para cada fase de utilización, sin importar cuáles fueran su composición por género, experiencia o estudios, pero sí la participación en la atención a los pacientes, de acuerdo con el IDH de los estados. Conclusiones. La herramienta se validó de modo interno y externo para su aplicación en diversas condiciones de México. Puede aplicarse en cualquier país de habla hispana.

Palabras clave: conocimiento; investigación en servicios de salud; administración de servicios de salud; apoyo a la planeación en salud; México
González-Block MA, Mercado FJ, Ochoa H, Rivera H, Idrovo AJ. Research utilization by health managers.

Validating a self-assessment tool.

Salud Publica Mex 2008;50:490-497.

\section{Abstract}

Objective.A tool was adapted and evaluated for the selfassessment by health management teams of their capacity to utilize research, distinguishing between its acquisition, analysis, adaptation and application. Material and Methods. The tool was translated into Spanish and a manual was created. The manual was submitted for authorization to the Ethics Committee of the National Public Health Institute of Mexico.The kit was sent to 122 units (response rate of $84.4 \%$ ), covering a wide range of institutions in six contrasting states of Mexico according to the Human Development Index (HDI). The tool was validated internally and externally using statistical methods. Results. Differences were observed in the teams' research utilization capacity within each phase, regardless of their composition by gender, experience or academic level but with differences according to the extent of involvement in patient care and to the states' HDI. Conclusions. The tool was validated both internally and externally for its application under widely varying conditions in Mexico. The tool can be applied in any Spanish speaking country.

Key words: knowledge; health services research; health services administration; health planning support; Mexico

(I) Centro de Investigaciones en Sistemas de Salud, Instituto Nacional de Salud Pública, México.

(2) Departamento de Salud Pública, Universidad de Guadalajara, México.

(3) Colegio de la Frontera Sur. México.

(4) Facultad de Medicina y Psicología, Universidad Autónoma de Baja California, México.

Fecha de recibido: 7 de enero de 2008 - Fecha de aceptado: 15 de julio de 2008 Solicitud de sobretiros: Dr. Miguel Angel González Block. Centro de Investigación en Sistemas de Salud, Instituto Nacional de Salud Pública. Av. Universidad 655, col. Santa María Ahuacatitlán. 62508, Cuernavaca, Morelos, México. Correo electrónico: mgonzalezblock@correo.insp.mx 
$\mathrm{E}^{\mathrm{n}}$ la actualidad, la investigación en salud experimenta una institucionalización gradual como factor de orientación y cambio en el sector salud en los países de ingresos medios y bajos. Prueba de ello son los esfuerzos por introducir la medicina y enfermería sobre bases científicas, la vigilancia participativa de la calidad, la comparación sistemática de resultados de desempeño y el aprendizaje compartido de experiencias de reforma a los sistemas de salud. La investigación estratégica, entendida como aquella cuyo conocimiento se enfoca en la resolución de problemas, intenta disminuir la gran variabilidad que caracteriza a la práctica médica y al desempeño de los servicios de salud en los ámbitos nacional e internacional; de esa forma es posible lograr mayor eficiencia, calidad y equidad en los recursos públicos. Para conferir congruencia a estas acciones es necesario el fortalecimiento de los sistemas nacionales de investigación en su conjunto, de tal manera que pueda incrementarse la capacidad de investigación al igual que el rápido y efectivo uso del conocimiento. ${ }^{1,2}$

La utilización de la investigación por los proveedores y directivos de servicios de salud ha recibido particular atención en relación con las medidas de fortalecimiento de los sistemas de investigación en salud. Se han analizado las disposiciones para mejorar la gestión del conocimiento dentro del sector salud y se han puesto en práctica programas de capacitación, tanto de investigadores en el proceso de difusión y diseminación como de usuarios en el proceso de demanda y aplicación. ${ }^{3,4}$ Estudios y revisiones sistemáticas aparecidos en las publicaciones sobre el proceso de utilización de la investigación han delineado la necesidad de establecer una relación temprana, intensa, personal, continua y de largo plazo entre los investigadores y los tomadores de decisiones para poner en práctica esfuerzos de promoción, interacción y demanda de investigación. ${ }^{5-9}$ Se ha descrito, asimismo, una gran variedad de interfaces para que los investigadores divulguen sus resultados, con objeto de que los usuarios los demanden y fomenten la interacción entre ambos. ${ }^{10,11}$

El fortalecimiento de las interfaces entre la investigación y los servicios de salud se ha identificado como una medida clave para lograr el aprovechamiento de los resultados de investigación. Para ello resulta fundamental diagnosticar la capacidad de los proveedores y directivos de servicios de salud para demandar de manera informada y utilizar con efectividad los resultados de investigación.

Para responder a estos retos, la Fundación Canadiense para la Investigación en Servicios de Salud diseñó en 2001 una herramienta de autodiagnóstico de la capacidad de utilización de la investigación. La herramienta se ha aplicado de manera amplia y se ha evaluado de modo positivo con base en 32 grupos focales y encuestas con 292 instituciones que la demandaron entre finales de 2005 y principios de $2006 .{ }^{4}$ De acuerdo con estos resultados, se consideró pertinente adaptar e introducir la herramienta de autodiagnóstico en países de habla hispana y de ingresos medios y bajos. Esta tarea se analizó en el Consorcio para la Investigación para el Desarrollo de los Sistemas Estatales de Salud (INDESES), con apoyo del CONACYT, cuyo grupo técnico está constituido por las instituciones autoras de este artículo. La herramienta se consideró útil para apoyar diversas disposiciones de fortalecimiento ideadas para promover la planeación estratégica para el fortalecimiento de la capacidad dentro de las organizaciones de servicios y el ámbito de las instituciones de salud en los planos estatal y nacional.

La aplicación de la herramienta por parte de INDESES tuvo dos objetivos fundamentales: por un lado, adaptar y validar la herramienta en el contexto mexicano; por el otro, apoyar la planeación estratégica con base en el fortalecimiento de la utilización de los resultados de investigación en cada organización participante. Este artículo se propone presentar y validar la herramienta de autodiagnóstico de la capacidad de uso de los resultados de investigación. El análisis del autodiagnóstico de la capacidad de utilización de resultados y la propuesta de estrategias de fortalecimiento se describen en otro artículo. $^{12}$

\section{La herramienta de autodiagnóstico}

La herramienta está diseñada como un apoyo para la planeación estratégica. Es por ello que se privilegia su autoaplicación por parte del equipo gerencial y se fomenta la búsqueda de consenso en torno de la capacidad del equipo. Puede utilizarse para establecer una situación basal o bien para evaluar los resultados de intervenciones de fortalecimiento.

La herramienta se compone de cuatro secciones y su aplicación no debe tomar más de 90 minutos. En la primera sección se identifican las características del equipo directivo participante. La sección II ("Diagnóstico") explora la capacidad de la organización en las cuatro "a": adquirir, analizar, adaptar y aplicar los resultados de la investigación. En la sección III ("Nuestras necesidades según los resultados") se identifican los objetivos para el fortalecimiento organizacional con base en los resultados del diagnóstico. La sección IV (“Qué sigue?") ayuda a formular preguntas y disposiciones para el fortalecimiento de las capacidades de la organización, a la vez que ayuda a identificar necesidades de apoyo técnico.

La herramienta se tradujo al español y se modificó su estructura para facilitar la aplicación. Algunas pre- 
guntas también se adaptaron para aclarar su sentido. Se mantuvieron todos los reactivos del original. La herramienta está disponible en la página electrónica del Instituto Nacional de Salud Pública (http: / / www.insp. $\mathrm{mx} /$ Portal/Centros/ciss/ciss_index.html).

La fase de adquisición del conocimiento explora los aspectos de estructura y procesos dedicados a la investigación. Los aspectos de estructura se enfocan en la suficiencia del personal capacitado, la dedicación de tiempo, los incentivos (cursos y talleres de capacitación, asistencia a congresos, estímulos o compensaciones adicionales, etc.), los recursos (conocimientos, financiamiento, equipo de cómputo, acceso a biblioteca, internet, etc.) y los convenios con expertos externos. Los procesos se centran en el acceso a revistas científicas, boletines, informes, bases de datos y páginas electrónicas. De igual modo, se explora la colaboración con investigadores, ya sea a través de reuniones informales, de acuerdo con el patrocinio de actividades específicas de investigación o bien a través de redes informales o formales para intercambiar ideas, experiencias y las mejores prácticas.

La fase de análisis de los resultados de investigación explora la capacidad para determinar si la investigación es confiable, pertinente, aplicable y de alta calidad. Para ello se determina la suficiencia de la estructura organizacional en términos del personal con habilidades y herramientas de análisis (p. ej., para identificar información relacionada y comparar sus métodos y resultados), así como la disponibilidad de convenios con expertos externos.

La fase de adaptación se enfoca en la capacidad para sintetizar resultados de investigación de forma comprensible para el usuario, además de comunicarla a la organización. Se describen aspectos de estructura, habilidades y colaboración externa para presentar los resultados de la investigación de manera concisa y en un lenguaje accesible; la finalidad es sintetizar en un solo documento toda la investigación relevante, vincular los resultados de la investigación con los problemas centrales que enfrentan los tomadores de decisiones y facilitar las decisiones sobre las acciones recomendadas en la investigación.

La fase de aplicación es la más amplia de todas y su hilo conductor es el liderazgo, con base en la investigación y la valoración y aplicación de la investigación a las decisiones de la organización. Se identifica en primer lugar la prioridad de la utilización de la investigación en términos de los recursos dedicados a esta tarea, la autonomía para decidir sobre cambios fundamentados en los resultados, la claridad de la estrategia para comunicar las necesidades de investigación y los valores que acompañan a la investigación en el proceso de tomar decisiones. Se describe a continuación el nexo de la investigación con el proceso de tomar decisiones, incluidas la identificación de preguntas de investigación, la consideración de hallazgos -tanto de investigaciones propias como externas- y la evaluación de la factibilidad de efectos de diversas soluciones basadas en resultados.

En esta sección se abordan también los retos de la aplicación de los resultados en cada organización, de manera concreta en torno de la claridad del proceso de tomar decisiones. Se analiza si el equipo conoce con claridad el modo y el momento de tomar decisiones importantes, aportar evidencias y emplearlas. Se reconoce en este contexto si el personal que proporciona y analiza resultados participa en las discusiones para adoptar decisiones y si se retroalimentan dentro de la organización las decisiones y su racionalidad con base en la investigación.

Una vez que el grupo directivo ha explorado su situación en relación con cada una de las cuatro a, se cuantifica la capacidad de utilización a partir de ocho dimensiones (cuadro I). Sobre esta base, el equipo concluye el autodiagnóstico y formula preguntas en formato abierto para orientar el diseño o bien la aplicación de programas de fortalecimiento de la capacidad.

El coordinador debe estimular la formulación de preguntas acerca de la manera de promover la importancia de la investigación dentro de la organización, la obtención de acceso a personal capacitado y apoyos externos, la disponibilidad de capacitación y acceso a referencias para la abogacía en favor de la investigación y, por último, los costos de adquisición de resultados y las fuentes de financiamiento disponibles, entre otras.

\section{Metodología para la validación}

La metodología se diseñó para validar la herramienta en los planos interno y externo, en el primero tras analizar la relación entre las diferentes secciones, subsecciones y reactivos, y en el segundo tras observar el vínculo entre la capacidad de cada unidad y su contexto institucional y socioeconómico. Para ello se llevó a cabo un estudio de tipo cuantitativo y cualitativo con una muestra de equipos de gestión de unidades operativas y conducción de programas en entidades con diferentes condiciones socioeconómicas. Se seleccionó a Baja California y Sonora en la región norte, Chiapas y Tabasco en la región sur y Jalisco y Zacatecas en la región centro-occidente.

Las unidades de servicio del sector salud público y privado se eligieron para incluir la atención preventiva y curativa ambulatoria, la atención hospitalaria, la gestión de programas jurisdiccionales y las instituciones públicas y privadas no lucrativas que ofrecen servicios de salud. Se concedió particular atención a la inclusión de unidades que suministran servicios de salud a 


\section{Cuadro I}

INDICADORES Y ESCALA DE MEDICIÓN PARA EL FORTALECIMIENTO DE LA CAPACIDAD DE UTILIZAR RESULTADOS DE INVESTIGACIÓN

I. La prioridad asignada a la investigación dentro de la organización debe ser mayor o igual.

2. La integración de la investigación debe iniciarse, incrementarse o mejorarse en su calidad.

3. La utilización de la investigación por los tomadores de decisiones es nula, ocasional o suficiente.

4. Para incrementar la capacidad de utilización de resultados se requiere aumentar, sobre todo, personal calificado, recursos financieros, tiempo, incentivos o convenios con expertos.

5. Para adquirir la investigación se necesita, en particular, contar con mayor acceso a revistas científicas, reportes, bases de datos, páginas de internet, colaboración con investigadores o aprendizaje de colegas.

6. Respecto de la evaluación de la investigación, se requiere introducirla, hacerlo con más frecuencia o mejorar su calidad.

7. En cuanto a las habilidades para la elaboración de resúmenes de investigación, es preciso desarrollarlas, aumentarlas o mejorarlas.

8. Para vincular los resultados de la investigación con los tomadores de decisiones se necesita iniciar su relación, hacerlo con más frecuencia o mejorar su calidad.

poblaciones vulnerables (pobres, ancianos, migrantes e indígenas), cuya atención con calidad y eficiencia requiere la utilización efectiva de resultados de investigación. La muestra incluyó asimismo la diversidad de instituciones prestadoras de servicios de salud. No se pretendió conformar una muestra representativa por tamaño e institución. La selección de unidades se basó en la racionalidad del muestreo teórico. ${ }^{13}$

Las unidades de estudio incluidas en cada estado se calcularon para no ser menores de ocho instituciones de salud públicas y ocho privadas no lucrativas. Las instituciones públicas en cada entidad fueron la Secretaría de Salud (población en el sector informal) y el Instituto Mexicano del Seguro Social (IMSS) (trabajadores y sus familiares del sector formal). En entidades seleccionadas se incluyó a unidades del programa IMSS-Oportunidades (población rural de predominio indígena), el Instituto de Seguridad Social para los Trabajadores al Servicio del Estado (ISSSTE), el Desarrollo Integral de la Familia (DIF) y Petróleos Mexicanos (PEMEX). Las instituciones de la sociedad civil se seleccionaron para incluir a aquellas que prestan servicios a grupos vulnerables de particular interés en cada entidad, con especial atención en individuos pobres, ancianos, indígenas y migrantes.

Para seleccionar las unidades de estudio se elaboró primero un censo de las instituciones y sus respectivos programas de salud en cada uno de los seis estados. Las unidades en el censo se clasificaron de acuerdo con institución, tipo de servicio y población atendida. Sobre esta base se efectuó una preselección de 20 unidades por estado, con atención a la necesidad de incluir la gama de instituciones en cada estado.

Se entabló contacto con los responsables de cada unidad seleccionada, sobre todo por vía telefónica, para concertar citas y explicar la mecánica del ejercicio. Dicho esfuerzo fue diferente según fuera la institución, con una mayor gestión central en el caso del IMSS. Durante estas reuniones se proporcionó una breve capacitación a la persona que coordinó la aplicación del instrumento para operarlo en una sesión grupal integrada con profesionales del equipo directivo o individuos que laboraban en la unidad de tiempo completo. Se determinó una sola sesión con duración de 60 a 75 minutos. El coordinador capacitado convocó a la reunión y entregó en el acto un ejemplar del instrumento a cada integrante del grupo para que se leyera y contestara de manera individual; a continuación se discutió en grupo y se alcanzó un consenso en todas las respuestas. El coordinador registró éstas en una copia del instrumento. Cuando no se consiguió el consenso en algún reactivo en particular, el coordinador anotó el promedio de los valores individuales. Para los fines de la validación, se solicitó al coordinador que remitiera al equipo de investigación una copia del instrumento.

Se realizó una descripción de las instituciones y principales características de los sujetos participantes. Luego se describieron las puntuaciones obtenidas para cada uno de los ítems del cuestionario y se compararon los obtenidos por las entidades federativas. Dado que varios equipos de gestión no contestaron algunas preguntas, y ello podía dificultar la comparación de las puntuaciones obtenidas, se exploró la correlación entre los diversos componentes del cuestionario mediante el cálculo de la ro de Spearman. Una vez verificada la correlación aceptable, se calcularon los puntajes para cada fase de todas las unidades; cada uno de estos correspondió a la sumatoria obtenida con las respuestas, dividido entre el total de respuestas sin datos faltante para cada fase. Este procedimiento posibilitó la inclusión de todas las unidades en los análisis subsecuentes, teniendo una misma escala para todas las fases. Se exploró la relación existente entre las puntuaciones medianas de cada entidad federativa con el Indice de Desarrollo Humano de 2004 respectivo, a través del cálculo de la ro de Spearman. Todos los análisis se llevaron a cabo con el programa estadístico Stata 9 (Stata Corporation, College Station, Texas). 


\section{Resultados}

\section{Características de las unidades, los profesionales y los equipos}

Se enviaron 122 cédulas a igual número de unidades de estudio. Se recibieron $103(84.4 \%)$ cédulas válidas, distribuidas de modo satisfactorio entre todas las entidades federativa, instituciones, tipos de servicio y grupos vulnerables incluidos en el estudio. Participó un total de 586 profesionales conformado con tomadores de decisiones y personal operativo, personal médico y no médico, profesionales con posgrado, así como hombres y mujeres. La antigüedad en el puesto fue de 5.23 años como mediana (cuadro II).

Intervinieron en promedio 5.7 personas por grupo. El cuestionario se llenó por una sola persona en 14 casos, ocho grupos reunieron entre dos y cinco personas, mientras que 15 grupos incluyeron a más de ocho personas. Las características de los grupos de autodiagnóstico muestran una composición similar, con valores extremos bajos en las variables individuales. Sólo 15.5\% de los grupos se integró de manera exclusiva con médicos, mientras que en $26.2 \%$ no se identificó la presencia de estos profesionales. Hasta 18.5\% de los grupos contó con la participación exclusiva de mujeres y $5.8 \%$ de hombres. Una proporción de $44.7 \%$ de los grupos tenía una participación mayoritaria de mujeres. En 13.7\% de los grupos había profesionales con promedios mayores de 10 años de antigüedad, mientras que en $14 \%$ sólo se notificó menos de dos años en promedio. En 18.6\% de los grupos todos los profesionales contaban con posgrado, mientras que en $29 \%$ ninguno de ello poseía esas calificaciones.

\section{Validación externa}

No se detectaron diferencias significativas entre la composición de los grupos y el autodiagnóstico de acuerdo con género, años de experiencia o estudios de posgrado $(p>0.05)$. Cuando hay menor participación de tomadores de decisión o médicos dentro del grupo se observa una mayor puntuación en el autodiagnóstico. No obstante, la participación de los médicos muestra un comportamiento ambiguo, ya que la puntuación del autodiagnóstico es mayor en grado significativo también cuando su proporción entre los participantes del grupo es muy alta.

Se observaron diferencias de relevancia estadística entre estados en los valores de las cuatro fases del proceso de utilización. También se identificaron diferencias de importancia estadística en la mayoría de los 40 ítems a través de los estados (cuadro III). Las excepciones son

\section{Cuadro II}

Principales características de las instituciones $(\mathrm{N}=$ I 03) E INDIVIDUOS PARTICIPANTES $(\mathrm{N}=586)$ EN EL ESTUDIO*

\begin{tabular}{lrr} 
Características personales & $n$ & $\%$ \\
$\quad$ Hombres & 296 & 50.5 \\
$\begin{array}{l}\text { Profesión } \\
\text { Médico }\end{array}$ & \\
\hline Enfermero & 306 & 52.2 \\
\hline Otro & 49 & 8.4 \\
\hline
\end{tabular}

Tipo de cargo

$\begin{array}{lll}\text { Tomadores de decisiones } & 257 & 43.9\end{array}$

Personal operativo $\quad 324 \quad 55.3$

\begin{tabular}{lrr} 
Sin datos & 5 & 0.8 \\
\hline
\end{tabular}

Estudios de posgrado

$261 \quad 44.5$

Experiencia (años promedio)

\begin{tabular}{|c|c|c|}
\hline Menos de 3 & 26 & 25.2 \\
\hline De 3 a menos de 5 & 22 & 21.4 \\
\hline De 5 a menos de 7 & 32 & 31.1 \\
\hline 7 o más & 23 & 22.3 \\
\hline \multicolumn{3}{|c|}{ Características institucionales } \\
\hline \multicolumn{3}{|l|}{ Entidad federativa } \\
\hline Baja California & 16 & 15.5 \\
\hline Chiapas & 22 & 21.4 \\
\hline Jalisco & 16 & 15.5 \\
\hline Sonora & 11 & 10.7 \\
\hline Tabasco & 20 & 19.4 \\
\hline Zacatecas & 18 & 17.5 \\
\hline
\end{tabular}

Grupo vulnerable atendido

\begin{tabular}{lll} 
Ancianos & 70 & 67.9 \\
\hline Indígenas & 54 & 52.4 \\
\hline Migrantes & 62 & 60.2 \\
\hline Pobres & 82 & 79.6
\end{tabular}

Tipo de servicio ${ }^{\ddagger}$

\begin{tabular}{lcc} 
Promoción de la salud & 70 & 68 \\
\hline Educación en salud & 63 & 61.2 \\
\hline Prevención de enfermedad & 84 & 81.6 \\
\hline Atención a enfermos & 77 & 74.8 \\
\hline Rehabilitación & 33 & 32 \\
Tipo de institución & & \\
Secretaría de Salud (estatal) & 35 & 34 \\
\hline IMSS & 10 & 9.7 \\
\hline IMSS-Oportunidades & 5 & 4.9 \\
\hline ISSSTE & 2 & 1.9 \\
\hline DIF & 5 & 4.9 \\
\hline OSC & 36 & 34.9 \\
\hline Otras privadas & 10 & 9.7
\end{tabular}

* Aplicación de la encuesta entre abril y noviembre del año 2007

₹ La suma es mayor de $100 \%$ debido a que varias instituciones atienden a más de una población u ofrecen más de un servicio

IMSS = Instituto Mexicano del Seguro Social

ISSSTE= Instituto de Seguridad y Servicios Sociales de los Trabajadores del Estado

DIF= Sistema para el Desarrollo Integral de la Familia

OSC = Organizaciones de la Sociedad Civil 
siete de los 12 ítems del componente de adquisición de la investigación y 12 de los 15 del de aplicación ( $p>0.05)$. No se encontraron diferencias al comparar los resultados entre instituciones participantes (cuadro IV).

\section{Validación interna}

Se reconocieron diferencias y correlaciones de significado estadístico entre todas las fases y subfases que componen el proceso de utilización de resultados de investigación. Ello significa que existe una correspondencia entre la mayor capacidad en una fase y subfase y el resto de ellas.

Los resultados muestran una diferencia significativa $(p<0.01)$ de la capacidad entre las cuatro fases de la utilización de resultados de investigación, sea en el plano nacional o en el interior de cada entidad federativa y entre instituciones, salvo en el caso del ISSSTE (cuadro III). Al analizar las diferencias entre pares de fases con la prueba de Scheffé se encuentran también diferencias significativas entre todas ellas, salvo entre análisis y adaptación, por un lado, y análisis y aplicación, por otro. En consecuencia, la fase de adquisición de resultados de investigación es la que mayores diferencias observa respecto de las demás.

La correlación entre procesos de utilización de resultados de investigación que muestra la mayor fuerza se reconoció en la fase de "Análisis de la información" entre la subfase "Capacidad para evaluar la confiabilidad y la calidad de la evidencia", por un lado, y la subfase "Determinación de la pertinencia y la aplicabilidad", por el otro, con una cifra de 0.77 . La segunda vinculación más fuerte (0.75) se observó en la fase "Aplicación de la investigación", entre la subfase de "Liderazgo con el ejemplo y valoración de la investigación" y la subsección "Lugar para la investigación en la toma de decisiones". La correlación sugiere que se fomentan mayores acciones para la aplicación cuanto mayor es la apertura dentro del equipo directivo para aplicar la investigación. Una tercera correlación que vale la pena enfatizar por su fuerza (0.72) se identifica entre las subfases que evalúan los extremos del proceso de utilización: la "Capacidad para la adquisición de resultados de investigación" y la subsección "Asignación de un lugar para la investigación en la toma de decisiones".

\section{Cuadro III}

Puntuaciones, mediana y CORRELACión en CADA faSe y entre entidad federativa e institución ( $\mathrm{N}=103$ )*

\begin{tabular}{|c|c|c|c|c|c|c|}
\hline & Adquisición & Análisis & Adaptación & Aplicación & Total & $p^{\ddagger}$ \\
\hline Todas las entidades federativas & 0.91 & 2.40 & 2.00 & 2.43 & 1.60 & $<0.01$ \\
\hline \multicolumn{7}{|l|}{ Entidad federativa } \\
\hline Sonora & 1.06 & 2.8 & 2.5 & 3.33 & 1.90 & $<0.01$ \\
\hline Tabasco & 1.02 & 2.8 & 2.29 & 2.86 & 1.79 & $<0.01$ \\
\hline Baja California & 1.05 & 2.6 & 2.19 & 2.43 & 1.66 & $<0.01$ \\
\hline Jalisco & 0.84 & 1.8 & 1.78 & 2.39 & 1.44 & $<0.01$ \\
\hline Chiapas & 0.75 & 2 & 1.76 & 2.35 & 1.27 & $<0.01$ \\
\hline Zacatecas & 0.75 & 1.5 & 1.65 & 1.86 & 1.26 & $<0.01$ \\
\hline Valor $p^{*}$ & $<0.01$ & $<0.01$ & $<0.01$ & $<0.01$ & $<0.01$ & $<0.01$ \\
\hline \multicolumn{7}{|l|}{ Tipo de institución } \\
\hline Secretaría de Salud (federal/estatal) & 0.88 & 2.60 & 2.18 & 2.40 & 1.60 & $<0.01$ \\
\hline IMSS & 1.03 & 2.50 & 1.96 & 2.55 & 1.67 & $<0.01$ \\
\hline IMSS-Oportunidades & 0.72 & 1.40 & 1.67 & 2.36 & 1.23 & $<0.01$ \\
\hline ISSSTE & 0.80 & 2.20 & 1.94 & 1.73 & 1.28 & 0.16 \\
\hline DIF & 0.81 & 1.60 & 1.82 & 1.86 & 1.25 & 0.03 \\
\hline ONG & 0.91 & 2.00 & 2.00 & 2.53 & 1.60 & $<0.01$ \\
\hline Otras privadas & 0.92 & 2.50 & 2.00 & 2.86 & 1.65 & $<0.01$ \\
\hline Valor $p^{*}$ & 0.34 & 0.20 & 0.67 & 0.61 & 0.78 & \\
\hline
\end{tabular}

* Aplicación de la encuesta entre abril y noviembre del año 2007

\# Prueba de Kruskal-Wallis

IMSS = Instituto Mexicano del Seguro Social

ISSSTE= Instituto de Seguridad y Servicios Sociales de los Trabajadores del Estado

DIF= Sistema para el Desarrollo Integral de la Familia 
Cuadro IV

Correlaciones de Spearman entre las diferentes puntuaciones obtenidas en las subescalas DEL CUESTIONARIO DE AUTODIAGNÓSTICO*
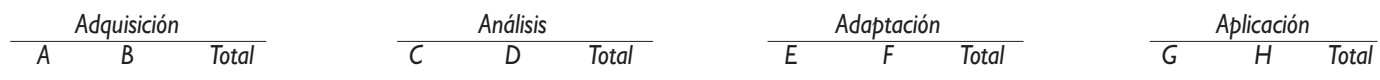

Adquisición

\begin{tabular}{llll} 
A & I & \\
\hline B & 0.58 & I & \\
\hline Total & 0.85 & 0.91 & I
\end{tabular}

Análisis

\begin{tabular}{lllllll}
$C$ & 0.62 & 0.62 & 0.67 & I & & \\
\hline D & 0.66 & 0.54 & 0.66 & 0.77 & I & \\
\hline Total & 0.67 & 0.63 & 0.71 & 0.95 & 0.91 & I
\end{tabular}

\begin{tabular}{llllllllll}
$\begin{array}{l}\text { Adaptación } \\
\mathrm{E}\end{array}$ & 0.56 & 0.46 & 0.56 & 0.67 & 0.68 & 0.72 & & $\mathrm{I}$ & \\
\hline $\mathrm{F}$ & 0.63 & 0.65 & 0.70 & 0.67 & 0.66 & 0.68 & 0.49 & $\mathrm{I}$ \\
\hline Total & 0.74 & 0.75 & 0.80 & 0.81 & 0.79 & 0.83 & 0.84 & 0.87 & $\mathrm{I}$
\end{tabular}

\begin{tabular}{|c|c|c|c|c|c|c|c|c|c|c|c|}
\hline G & 0.55 & 0.62 & 0.65 & 0.64 & 0.63 & 0.68 & 0.64 & 0.54 & 0.71 & I & \\
\hline $\mathrm{H}$ & 0.72 & 0.63 & 0.70 & 0.53 & 0.65 & 0.60 & 0.61 & 0.61 & 0.69 & 0.75 & I \\
\hline Total & 0.73 & 0.70 & 0.76 & 0.59 & 0.68 & 0.66 & 0.64 & 0.69 & 0.77 & 0.89 & 0.96 \\
\hline
\end{tabular}

* Aplicación de la encuesta entre abril y noviembre del año 2007. Todas con $p<0.05$

A: personal y recursos (preguntas I-5); B: búsqueda de publicaciones y colaboración (preguntas 6-I2); C: capacidad de personal para evaluar la calidad de la evidencia (preguntas 13-I5); D: personal capacitado para evaluar la pertinencia (preguntas I6-I7); E: personal capacitado para sintetizar y adaptar los resultados (preguntas 18-2I); F: personal capacitado para sintetizar y adaptar los resultados (preguntas 22-25); G: acciones para la utilización de la investigación (preguntas 26-3I); H: papel de la investigación en las decisiones (preguntas 32-40)

La fase de aplicación del conocimiento tiene la mayor capacidad, con una mediana de 2.43. La adquisición de la investigación es la capacidad con menor fortaleza, con una mediana de 0.91 , un valor correspondiente a cerca de 33.5\% de la puntuación asignada a la fase de aplicación del conocimiento.

\section{Discusión}

La herramienta de autodiagnóstico de la capacidad de utilización de la investigación la aplicaron los equipos de gestión bajo estudio sin importar cuáles fueran el nivel socioeconómico de la entidad o la institución de salud. No obstante, la aplicación de la herramienta se relacionó con el esfuerzo del Consorcio INDESES para introducirla en la práctica institucional. Es de esperar una amplia aplicación si las autoridades nacionales o estatales adoptan la herramienta como parte de una medida de fortalecimiento institucional.
La investigación permitió validar la herramienta de manera interna y externa. En el primer caso, la herramienta discrimina a equipos de gestión con diferentes grados de capacidad en cada una de las cuatro fases del proceso de utilización de la investigación, si bien la fase de adquisición se diferenció con mayor claridad. En la validación que llevó a cabo CHSRF en Canadá se encontraron resultados similares, dado que en ese país la herramienta diferencia entre instituciones con alta y baja capacidad de adquisición y análisis de la investigación, aunque no es tan clara la diferenciación de las fases de adaptación y aplicación.

La validación externa se confirma al encontrar un nexo entre el grado de capacidad y los diferentes contextos socioeconómicos. Esta diferencia es similar a la reconocida en el desempeño de los sistemas de salud a través de la variabilidad socioeconómica de México. ${ }^{14}$ Las diferencias entre unidades de salud pertenecientes a la misma institución pueden explicarse por la sólida 
influencia que ejerce el contexto socioeconómico sobre la capacidad institucional, a pesar de las diferencias que pueden tener en sus propios recursos.

\section{Conclusiones}

La herramienta de autodiagnóstico de la capacidad de utilización de resultados de investigación probó su validez interna y externa para el contexto mexicano. La herramienta fue sensible a las diferencias socioeconómicas entre entidades. Asimismo, la herramienta probó ser de fácil aplicación y contó con una alta tasa de respuesta en el contexto del proyecto de validación.

Puesto que la herramienta ya se validó en Canadá, esta investigación sugiere que la herramienta puede aplicarse en países con muy diversas condiciones socioeconómicas. La herramienta se aceptó también en instituciones con estructuras, tamaños y grados de centralización distintos. No obstante, falta aún determinar el grado al que la aplicación de la herramienta puede mejorar el proceso de utilización de la investigación para tomar decisiones por parte de los equipos gerenciales. Ello dependerá de circunstancias diversas, como el interés y la capacidad del equipo gerencial y las autoridades institucionales para aprovechar la investigación.

Parece evidente la importancia de investigar los procesos de utilización de la evidencia en las decisiones de los equipos gerenciales y unidades de salud, bajo diferentes contextos socioeconómicos, inserción institucional y composición profesional. De manera más específica, es recomendable diseñar intervenciones para mejorar la utilización de la evidencia con base en las diferentes capacidades diagnosticadas con la herramienta aquí evaluada y la evaluación de los procesos y resultados.

Se recomienda la aplicación de la herramienta en diferentes niveles. Los órganos a cargo de fomentar la investigación dentro de las instituciones nacionales pueden promover su aplicación como un apoyo a la planeación estratégica de las unidades de servicio. Pueden, asimismo, aplicar la herramienta para elaborar diagnósticos institucionales que permitan asignar apoyos para el fortalecimiento de la capacidad de utilización de la investigación. La herramienta puede ser así el punto de partida para identificar indicadores específicos de fortalecimiento de la utilización de la investigación, además de robustecer los vínculos con las instituciones académicas y de investigación.

\section{Agradecimientos}

Esta investigación fue posible gracias al financiamiento del Fondo Sectorial de Investigación en Salud de CONACYT No. 14441. Los autores agradecen el apoyo brindado por los integrantes del Consejo Consultivo del Consorcio de Investigación para el Desarrollo de los Sistemas Estatales de Salud (INDESES). Se agradece asimismo la colaboración para el trabajo de campo de Jéssica Rivera Luna, Irma Xóchitl Fuentes Uribe, Rosario García y Víctor Becerril Montekio.

\section{Referencias}

I. Sadana R, Pang T. Health research systems: a framework for the future. Bull World Health Organ 2003;81:I59.

2. Stokes D. Pasteur's quadrant: basic science and technological innovation. Washington DC: Brookings Institution Press, 1997.

3. CTP Collaborative Training Program for Research for Policy and Action. Geneva:Alliance for Health Systems Research, 2004.

4. CHSRF Canadian Health Services Research Foundation. Is Research Working for You? Disponible en: http://www.chsrf.ca/other_documents/ working_e.php

5. Lomas J. Improving research dissemination and uptake in the health sector: beyond the sound of one hand clapping. Hamilton, Toronto: Centre for Health Economics and Policy Analysis, McMaster University, 1997.

6. Lomas J. Using "linkage and exchange" to move research into policy at a Canadian Foundation. Health Affairs 2000; 19:236-240.

7. Innvaer S,Vist G, Trommald H, Oxman A. Health policy-makers' perceptions of their use of evidence: a systematic review.J Health Serv Res Policy 2002;7:239-244.

8. Davies H, Nutley S. Healthcare: evidence to the fore. In:What Works? - Evidence-based policy and practice in public services. Davies HTO, Nutley SM, Smith PC eds. Bristol:The Policy Press, 2000:43-67.

9. Trostle J, Bronfman M, Langer A. How do researchers influence decision-makers? Case studies of Mexican policies. Health Policy Plan 1999; 14:103-114.

I0. Lavis JN, Ross SE, Hurley JE, Hohenadel JM, Stoddart GL, Woodward $\mathrm{CA}$, et al. Examining the role of health services research in public policymaking. Milbank Q 2002;80:I25-154.

I I. Hanney SR, Gonzalez-Block MA, Buxton MJ, Kogan M. The utilization of health research in policy making: concepts, examples and methods of assessment. Health Res Pol Sys 2003; I:2.

12. González Block MA, Mercado FJ, Ochoa H, Rivera H, Idrovo AJ. Utilización de investigación por gestores de salud en México: diagnóstico de la capacidad y propuestas de fortalecimiento. Salud Publica Mex 2008:50:498-507.

13. Kuzel AJ. Sampling in qualitative inquiry. In: Crabtree B, MillerW (eds.). Doing qualitative research. Newbury Park: SAGE, 1992.

14. Secretaría de Salud. Salud: México 200I-2005. Información para la rendición de cuentas. México: SSA, 2006. 